\title{
Digital Libraries as Phenotypes for Digital Societies
}

\author{
Gary Marchionini \\ University of North Carolina at Chapel Hill, USA \\ march@ils.unc.edu
}

\section{Extended Abstract}

The research and development community has been actively creating and deploying digital libraries for more than two decades and many digital libraries have become indispensable tools in the daily life of people around the world. Today's digital libraries include interactive multimedia and powerful tools for searching and sharing content and experience. As such, digital libraries are moving beyond personal intellectual prostheses to become much more participative and reflective of social history. Digital libraries not only acquire, preserve, and make available informational objects, but also invite annotation, interaction, and leverage usage patterns to better serve patron needs. These various kinds of usage patterns serve two purposes: first, they serve as context for finding and understanding content, and second, they themselves become content that digital libraries must manage and preserve. Thus, digital library research has expanded beyond technical and informational challenges to consider new opportunities for recommendations, support of affinity groups, social awareness, and cross-cultural understanding, as well as new challenges related to personal and group identity, privacy and trust, and curating and preserving ephemeral interactions. This trend makes digital libraries cultural institutions that reveal and hopefully preserve the phenotypes of societies as they evolve. This talk will illustrate this theoretical perspective with examples from our experience with the Open Video Digital Library over the past decade and with recent extensions (VidArch Project) that harvest YouTube video as a strategy for preserving cultural context for digital collections. 